\title{
Noradrenaline Enhances Temporal Auditory Contrast and Neuronal Timing Precision in the Cochlear Nucleus of the Mustached Bat
}

\author{
Manfred Köss| ${ }^{\mathrm{a}}$ and Marianne Vater \\ Zoologisches Institut, 8000 München 2, Federal Republic of Germany
}

In the mustached bat, Pteronotus parnellii, noradrenaline (NA) was applied lontophoretically to single units in the anteroventral cochlear nucleus. NA suppressed tonic components of auditory responses and enhanced phasic onset responses to pure tone stimuli. The enhancement of onset activity was most pronounced in awake bats and was due to a decrease in the latency jitter of the first tone-evoked spikes from $0.55 \mathrm{msec}$ in controls to $0.28 \mathrm{msec}$ during NA application. In addition, NA reduced spontaneous neuronal activity. Noradrenergic antagonists suppressed phasic onset activity and increased the latency jitter of onset spikes. Opposite to the effect of NA, the tonic response component increased during application of the $\beta$-antagonist propranolol but decreased during injection of the $\alpha_{1}$-antagonist corynanthine. Other putative transmitter substances tested, nonselectively depressed both phasic and tonic response components (GABA, glycine) or increased both components either similarly or had more pronounced effects on the tonic response components (ACh, glutamate). Thus, NA specifically enhances auditory temporal contrast in favor of transients and improves neuronal timing precision, which may be of relevance for auditory tasks like passive sound localization, echolocation, and recognition of temporal patterns.

The mammalian brain features a widespread noradrenergic system which mainly originates from the locus coeruleus (Moore and Bloom, 1979; Foote et al., 1983). Noradrenaline (NA) is thought to modulate neuronal processing during orienting behavior, vigilance, and stress (Foote et al., 1980; Aston-Jones and Bloom, 1981; Abercrombie and Jacobs, 1987). In various brain areas which receive noradrenergic terminals, exogenously applied NA has either excitatory or inhibitory effects on the level of spontaneous neuronal activity (in vitro: Basile and Dunwiddie, 1984; Collins et al., 1984) and enhances both excitatory and inhibitory responses caused by iontophoretic application of GABA, glutamate, and ACh (Moises et al., 1979; Waterhouse et al., 1980) or by natural somatosensory (Waterhouse and Woodward, 1980) and auditory stimulation (Foote et al., 1975). To study whether NA has specific effects on the temporal processing of sensory information, we recorded auditory neurons in the anteroventral cochlear nucleus and the adjacent marginal

Received Jan. 31, 1989; revised May 31, 1989; accepted June 2, 1989.

We thank D. Caspary for familiarizing us with iontophoretic techniques and G. Klump. C. Müller, G. Neuweiler, W. Singer, and M. Zeise for critical comments on earlier versions of this manuscript. This work was supported by SFB 204

Correspondence should be addressed to Manfred Kössl at his present address: M.R.C. Neurophysiology Group, School of Biology, University of Sussex, Falmer, Brighton BN19QG, U.K.

Copyright $\odot 1989$ Society for Neuroscience $0270-6474 / 89 / 124169-10 \$ 02.00 / 0$ cell area of the echolocating bat, Pteronotus parnellii. Pteronotus emits multiharmonic echolocation calls and the dominant second harmonic consists of a constant-frequency (CF) component at about $60 \mathrm{kHz}$ followed by a frequency-modulated (FM) component sweeping down to $48 \mathrm{kHz}$ (Schnitzler, 1970). In the auditory cortex of Pteronotus the neuronal responses to the emitted pulse and returning echo are used to compute the time interval between pulse and echo, and therefore target range (O'Neill and Suga, 1979). Like most other auditory nuclei in Pteronotus, the CN is hypertrophied (Zook and Casseday, 1982, 1985). A distinct subregion of the anteroventral division of the $\mathrm{CN}$ and the marginal cell area receive the densest catecholaminergic innervation reported for the mammalian auditory system (Kössl et al., 1988), originating probably from the locus coeruleus. Presumed noradrenergic mechanisms involved in auditory orientation were expected to be well pronounced in this echolocating bat, since Pteronotus primarily depends on auditory information for spatial orientation and for locating insect prey.

\section{Materials and Methods}

Putative transmitter substances were injected microiontophoretically with balanced injection currents at 201 single units recorded in the anteroventral cochlear nucleus and the adjacent marginal area using 5-barreled glass pipettes with 6-10 $\mu \mathrm{m}$ outer tip diameter (Havey and Caspary, 1980) and a Neurophor-BH2 (WPI) iontophoretic injection device. The fine-tipped recording glass micropipette protruded $40-60$ $\mu \mathrm{m}$ beyond the multibarrel and was filled with $1.5 \mathrm{M} \mathrm{KCl} \mathrm{(6-10} \mathrm{M} \Omega$ ). The following substances were tested: NA $(0.2 \mathrm{M} l$-noradrenaline- $\mathrm{HCl}$, $\mathrm{pH} 4.5$; holding current, $-7 \mathrm{nA}), \mathrm{GABA}(0.5 \mathrm{~m}, \mathrm{pH} 3.8$; holding current, $-15 \mathrm{nA}$ ), glycine (Gly, $0.5 \mathrm{M}, \mathrm{pH} 3.8$; holding current, $-15 \mathrm{nA}$ ), bicuculline- $\mathrm{HCl}(0.005 \mathrm{M}, \mathrm{pH} 3.0$; holding current, $-15 \mathrm{nA})$, strychnine $(0.01 \mathrm{M}, \mathrm{pH} 3.5$; holding current, $-15 \mathrm{nA}), \mathrm{ACh}$ chloride $(0.5 \mathrm{M}, \mathrm{pH}$ 4; holding current, $-7 \mathrm{nA}$ ), l-glutamate (Glu, $0.2 \mathrm{~m}, \mathrm{pH} 8$; holding current $+15 \mathrm{nA}$ ), yohimbine- $\mathrm{HCl}(5 \mathrm{mM}, \mathrm{pH} 4.9$, holding current, -15 $\mathrm{nA}$ ), corynanthine- $\mathrm{HCl}$ (Cory, $5 \mathrm{mM}, \mathrm{pH} 4.9$; holding current, $-15 \mathrm{nA}$ ) l-dopamine- $\mathrm{HCl}(0.2 \mathrm{M}, \mathrm{pH} 4.5$; holding current, $-7 \mathrm{nA})$, and propranolol- $\mathrm{HCl}$ (Pro, $10 \mathrm{mM}, \mathrm{pH} 5.5$; holding current, $-15 \mathrm{nA}$ ). All drugs were obtained from Sigma. The specificity of the observed drug effects was verified by applying isotonic saline of the same $\mathrm{pH}$ as the drug solution and using the same current.

Seven mustached bats, $P$. parnellii, captured in Jamaica, weighing between 11 and $14 \mathrm{gm}$, were used for this study. During the initial surgery, in order to mount a small headholding metal bar on the skull, the animals were anesthetized with $3 \%$ halothane. After recovery from surgery, recording sessions of up to $3 \mathrm{hr} / \mathrm{d}$ took place over a period of 2-4 weeks. During recording the bats were anesthetized with $4 \mathrm{mg}$ Rompun $+25 \mathrm{mg}$ Ketanest $/ \mathrm{kg}$ body weight or stayed awake under local anesthesia with lidocaine. Experiments under anesthetized versus awake conditions were done in the same bats at different days. The temperature in the recording chamber was kept between 28 and $30^{\circ} \mathrm{C}$. The bats rested in a padded holder and frequently were allowed to drink during a recording session. Recording and analysis procedures followed those described by Feng and Vater (1985). The electrode was introduced through a small hole in the skull above the cerebellum according to stereotactic 


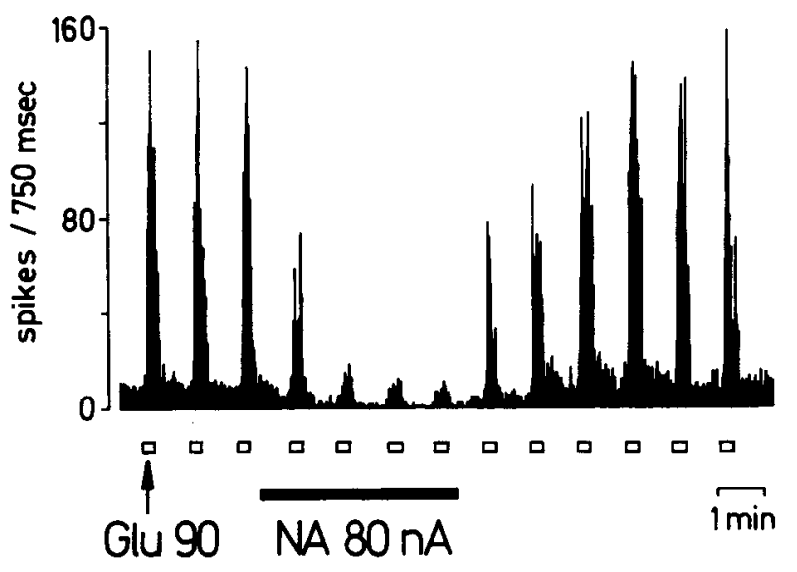

Figure 1. Continuous chart recording of spike activity of an auditory unit in the cochlear nucleus. The neuron was stimulated by pure-tone pulses of $30 \mathrm{msec}, 31 \mathrm{kHz}, 50 \mathrm{~dB} \mathrm{SPL}$, with a repetition period of 750 msec. NA depresses both normal activity and excitatory bursts induced by glutamate (Glu); open bars indicate periods of glutamate application.

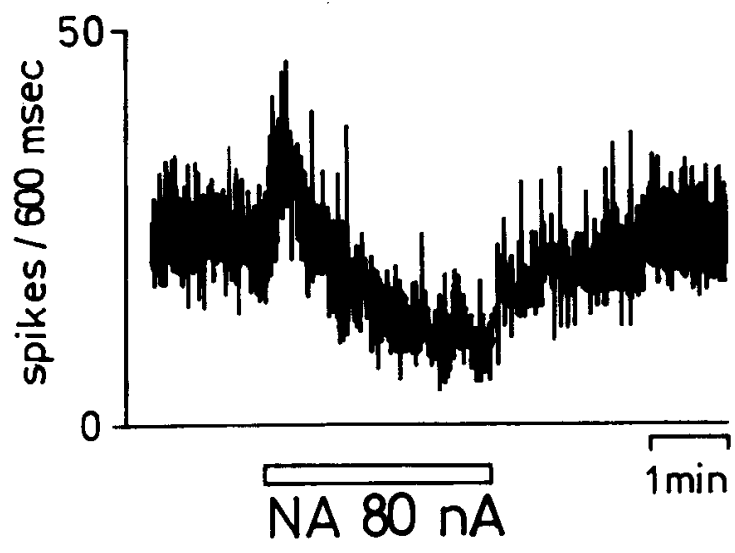

Figure 2. Chart recording of NA-induced changes in the overall spike activity of a single unit which was spontaneously active and responded to pure tones of $30 \mathrm{msec}, 22 \mathrm{kHz}, 65 \mathrm{~dB} \mathrm{SPL}$, with a repetition period $600 \mathrm{msec}$. After an initial increase, the activity was progressively depressed by NA. coordinates in relation to the metal bar. The recording sites in the cochlear nucleus were marked by electrolytic lesions or by injections of HRP. Pure-tone stimuli of 20-30 msec duration (rise/fall time, $1 \mathrm{msec}$ ) were applicd with the loudspeaker at $15 \mathrm{~cm}$ distance to the ipsilateral ear. The frequency of the stimulus was adjusted to the best frequency, i.e., the frequency of lowest threshold, of the individual neurons with the stimulus level usually between 20 and $50 \mathrm{~dB}$ above threshold (except Fig. 11). Neurons which were lost before recovery of the drug effects were discarded from the sample, as well as neurons which were recorded during penetrations after which the recording electrode was slightly separated from the injection pipette ( $<10 \%$ of all penetrations). Neuronal activity was monitored by continuous chart recordings. The width of the counting window corresponded to the repetition period of the acoustic stimuli $(0.5-1 \mathrm{sec})$. Therefore, each chart recorder point gives the sum of spontaneous spikes and the spikes elicited by a single-tone stimulus. In addition, 32-64 presentations of the same stimulus were used to construct poststimulus-time (PST) histograms with a bin width of $200 \mu \mathrm{scc}$. PST histograms were usually recorded between $30 \mathrm{sec}$ and 2 min after onset of the drug application. Bin widths from 100 to 400 $\mu \mathrm{sec}$ showed the same qualitative results, verifying that the effects of NA on the pattern of neural activity, as seen in PST histograms, was not an artefact of our choice of bin width. The statistical significance of differences between control data and data obtained during drug injection was analyzed using the Wilcoxon matched-pairs test (Siegel and Castellan, 1988).

\section{Results}

\section{Effects of NA on the level of neuronal activity}

NA depressed the level of general activity in single units. Figure 1 shows that both the basal activity level and excitatory bursts caused by pulsed injections of glutamate largely decreased during the application of NA. After offset of NA application, the activity level slowly recovered. Brief periods of excitation were occasionally observed at the onset of NA application (Fig. 2) or after offset. NA is reported to induce excitation at low concentrations and cause inhibition at higher concentrations (Basile and Dunwiddie, 1984). In our case, lowering the injection current or the concentration of NA in the glass pipette did consistently result in a decrease of the inhibition; however, the extent or duration of excitation did not change. Obviously, additional factors like the spatial relation between the tip of the injection pipette and the neuronal dendrites seem to be relevant for the degree of the observed excitation and inhibition.

\section{Effect of NA on auditory response patterns}

Most neurons tested with NA responded to pure-tone pulses with a phasic/tonic discharge pattern (163 units, Figs. $3, A, D$; $4 A$ ). NA differentially affected phasic and tonic response components. The phasic response to the stimulus onset was enhanced, and the tonic response to the ongoing pure tone was suppressed. Furthermore, spontaneous activity was reduced (Figs. $3 A, 4 A$ ). Of the population of units which exhibited on-enhancement, 25 units were analyzed in detail and showed a significant increase $(p>0.99)$ in the height of the onset peak by $57 \pm 34 \%$. This enhancement was based on 2 effects:

1. Under the influence of NA the latency of the first toneevoked spikes in relation to the onset of the tone stimulus was less variable (Fig. 4). The latency jitter, i.e., the average SD of latency, was reduced from $0.55 \pm 0.30 \mathrm{msec}$ in control and recovery measurements to $0.28 \pm 0.20 \mathrm{msec}$ during NA application (Fig. 5). The difference between the control and the NA condition was significant with $p>0.99$. The average latency did not change significantly under application of NA $(p=0.02$, difference between control and NA condition: $0.12 \pm 0.17 \mathrm{msec}$ ).

2. The number of spikes during the first $3 \mathrm{msec}$ of the response did not decrease as during the tonic part of the response but, on average, stayed constant. Application of NA decreased the number of spikes in the tonic response significantly by $43 \pm$ $37 \%(p>0.99)$, whereas there was no significant change within the first $3 \mathrm{msec}(p<0.01$, NA condition: $105 \pm 17 \%$ of control values). Onset enhancement was also commonly found in cases where the number of spikes during the first $3 \mathrm{msec}$ actually decreased. This indicates that the decrease in the latency jitter is the major source for enhancement of the onset peak.

The observed effects depended on the injection current (Fig. $6 A$ ) and the duration of delivery of NA (Fig. 6B). An increase in the level of the injection current from 10 to $100 \mathrm{nA}$ (Fig. $6 \mathrm{~A}$ ) caused a continuing decrease in the total number of spikes, whereas the height of the onset peak remained relatively stable over all currents. Thus, the onset enhancement seems to be rather independent of the general inhibitory effect of NA and can be elicited with very low injection currents. Differential 


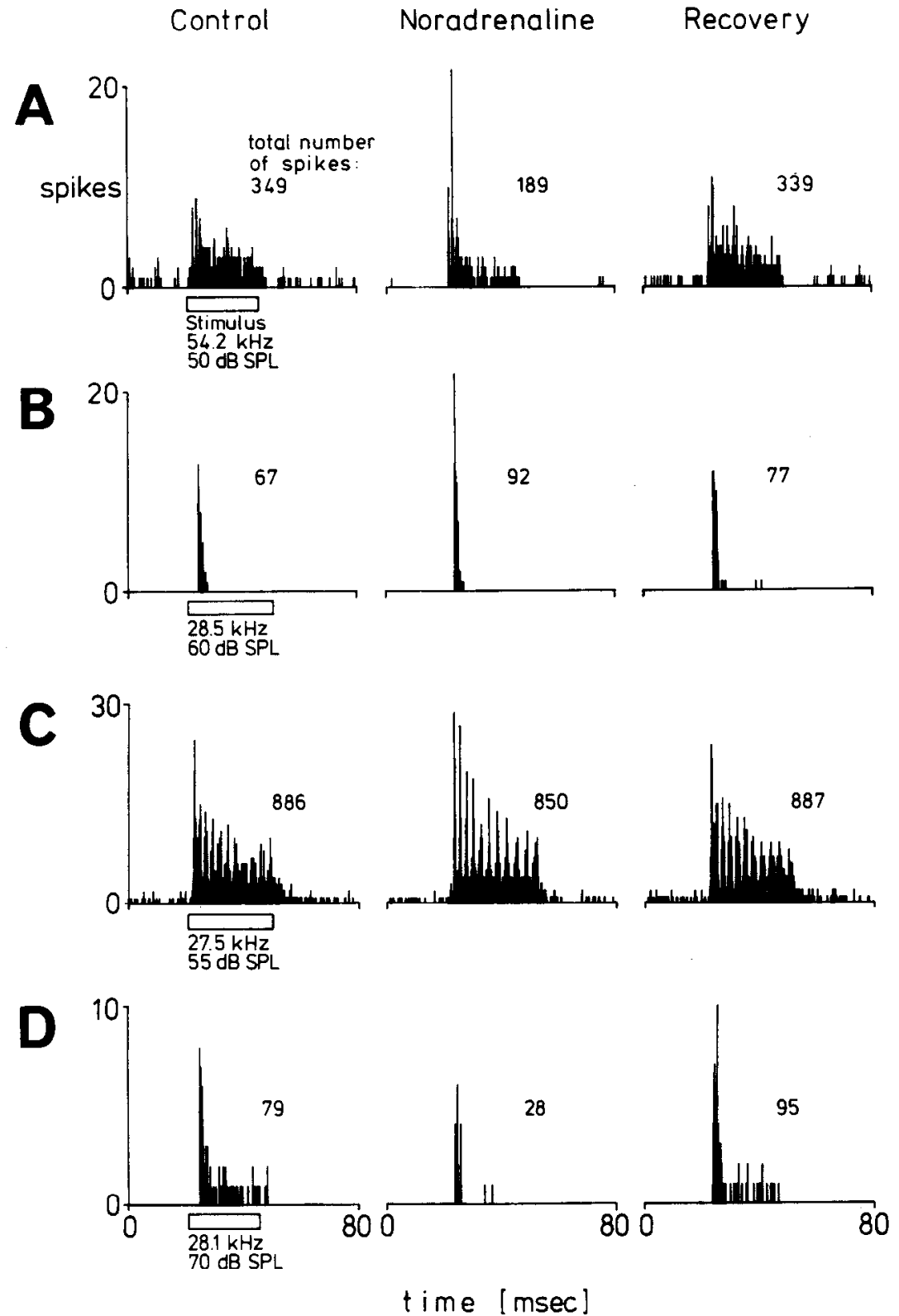

Figure 3. Examples of changes in auditory response patterns (PST histograms) of different single units, induced by $\mathrm{NA}$ in awake animals (70-90 nA injection current, 40 stimulus presentations, histogram bin width of $200 \mu \mathrm{sec}$, total number of spikes is given to the right of each histogram; the tone stimulus is indicated in the left column of histograms). $A$, Enhancement of auditory temporal contrast due to an increase of the phasic response component and a decrease of tonic response components and spontaneous activity. $B$, Enhancing action of NA on phasic on-responses. $C$, NA sharpens response peaks in a chopper neuron. $D$, Strong inhibition of both phasic and tonic components. effects of NA on the phasic/tonic response, i.e., an onset enhancement together with suppression of the tonic component, were most dramatic within the first 2 min of NA application (Fig. 6B), and maximum effects were elicited by injection currents between 50 and $100 \mathrm{nA}$ (Fig. 6A). The effects of NA did not depend on the best frequency of the neurons; NA equally affected neurons within the range of best frequencics encountered (11.7-96 kHz). In contrast to glutamate (see below), NA did not lower the neuronal thresholds to tone stimulation (4 neurons tested).

Apart from the prevailing phasic/tonic responders, we also recorded 4 purely phasic (Fig. 3B) and 2 chopper units (Fig. $3 C$ ). The latter responded to pure tones by a sequence of spikes with constant spike intervals. In the purely phasic units, NA again improved latency precision and also increased the number of elicited spikes. In chopper units, NA minimized the variability of the spike intervals, which resulted in sharper peaks of the chopper discharge pattern accompanied by a slight decrease in the total number of spikes.

Onset enhancements together with inhibition of the tonic response component were observed in $63 \%$ of all phasic/tonic responders in the awake animal. In $16 \%$ of the neurons, the inhibitory influence of NA dominated and the phasic response component was also depressed (Fig. $3 D$; Table 1). Cases of pure inhibition were seen frequently after prolonged application of NA (Figs. $6 B, 12$, normally after more than 2 min of injection).

\section{Effect of NA antagonists}

Propranolol, a $\beta$-antagonist, increased the overall activity and changed auditory response patterns in a way opposite to NA: in 8 of 10 neurons tested, iontophoretically applied propranolol specifically increased the tonic response component, whereas the onset peak was reduced, and the occurrence of the first tone evoked spike was desynchronized (Fig. $4 B$ ). This indicates that 

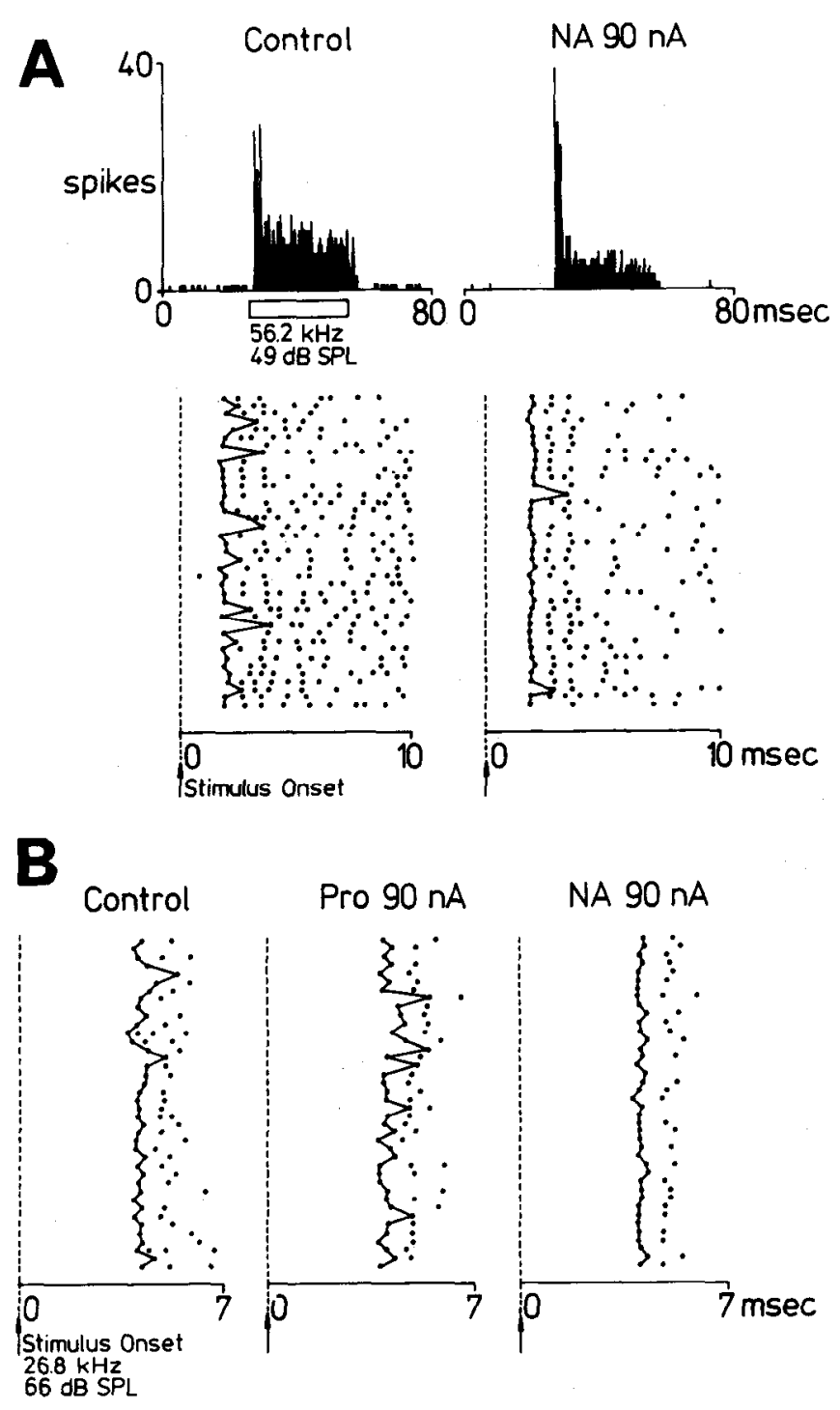

Figure 4. A, PST histogram (top row) and dot display (bottom) of the response of a single cochlear nucleus unit prior to and during application of NA. The PST histogram shows that the phasic response component is enhanced and the tonic response depressed by NA. Each row of the dot display represents the individual spikes to one stimulus, whose onset is given by the dashed line. The latencies of the first tone-evoked spikes (interconnected by straight lines) show far less jitter during application of NA than in the control. $B$, Comparison of the effects of NA and the $\beta$-antagonist propranolol: NA improves and propranolol destabilizes timing precision of onset spikes.

the action of endogenous NA is similar to that of NA injected iontophoretically. Further indication of the involvement of a $\beta$-adrenergic mechanism comes from the observation that dopamine, a $\beta$-agonist (Malenka and Nicoll, 1986), is able to produce onset enhancement coupled to tonic inhibition ( 2 of 8 neurons tested). Applied simultaneously with exogenous NA, propranolol reduced both onset enhancement and tonic inhibition caused by NA in 3 out of 8 neurons. In the remaining 5 neurons, propranolol could not block the effects of exogenous NA. $\alpha$-Antagonists (yohimbine- $\mathrm{HCl}$, corynanthine- $\mathrm{HCl}$ ) exhibited direct inhibitory effects. They suppressed the overall spike rate and decreased both phasic and tonic components of the auditory response ( 3 neurons tested for each antagonist). Ap-

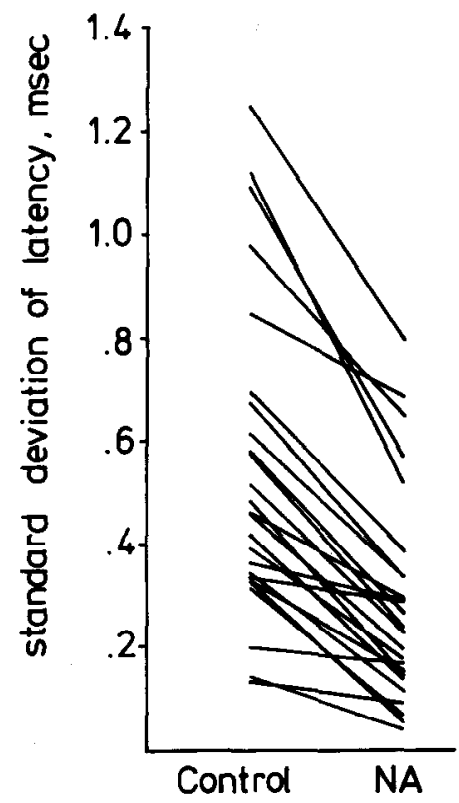

Figure 5. Temporal jitter of the onset spikes expressed as SD of latency. Each line connects the values obtained in a single unit. Application of NA narrows the range of temporal jitter (see text).

plication of the $\alpha_{1}$-antagonist corynanthine potentiated the overall inhibitory effect of exogenous NA and in 1 of 3 neurons tested could prevent onset-enhancement by simultaneously injected NA (Fig. 7). The range of best frequencies of neurons affected by either $\alpha$ - or $\beta$-antagonists was between 16.7 and $60.27 \mathrm{kHz}$. The effects were most pronounced for neurons tuned to frequencies between 20 and $30 \mathrm{kHz}$.

\section{Influence of anesthesia}

Under Rompun/Ketanest anesthesia, NA showed effects similar to that in awake animals. However, the proportion of units in which NA induced purely inhibitory effects, suppressing both the onset peak and the tonic discharge, increased considerably to $46 \%$ (16\% in awake bats), whereas differential on-enhancement was found in only $30 \%$ (63\% in awake bats). The anesthetics thus shift the range of action of NA towards inhibition.

\section{Effect of other transmitters on phasic/tonic response components}

To test if NA-induced enhancement of phasic and suppression of tonic activity is an effect specific to this transmitter or can be evoked by other neuroactive agents, we examined a variety of putative neurotransmitters for their influence on temporal response patterns.

\section{GABA and glycine}

GABA and glycine are known to act as inhibitory transmitters in the cochlear nucleus (Caspary et al., 1979). In Pteronotus, application of GABA $(n=26)$ or glycine $(n=17)$ suppressed the overall neuronal activity. In contrast to NA, in the vast majority of units both phasic and tonic components of auditory responses were equally affected (Figs. 8, 11; Table 1). Only in one unit, low injection currents of GABA differentially decreased the tonic component, while the onset peak remained constant. During application of the inhibitory transmitters, the 
jitter of latency increased significantly from 0.68 to $1.11 \mathrm{msec}$ (GABA, $p>0.99, n=15$ ) and from 0.53 to $0.93 \mathrm{msec}$ (glycine, $p>0.97, n=10)$. GABA significantly increased the latency by $0.58 \mathrm{msec}(p>0.99)$, whereas during injection of glycine, there were no significant changes in latency (increase by $0.09 \mathrm{msec}$, $p=0.18$ ). Of 16 units tested, 13 units were inhibited both by GABA and glycine, but not necessarily to a similar extent. For example, the unit in Figure 8 was much more sensitive to glycine. The amount of glycine released by pure diffusion (holding current switched off) was enough to inhibit the auditory response substantially (Fig. 8). The inhibitory effects could be blocked selectively with the GABA-A antagonist bicuculline $(n=4)$ and the glycine antagonist strychnine $(n=3)$.

\section{$A C h$}

Similar to NA, ACh is known to exert both excitatory and inhibitory effects (McGormick and Prince, 1987; Müller, 1987) and to modulate neuronal excitability (Brown, 1983; Müller, 1987). In the cochlear nucleus of the chinchilla, $\mathrm{ACh}$ is reported to have excitatory effects in the ventral and inhibitory effects in the dorsal subdivision (Caspary et al., 1983). Our study was restricted to the ventral subdivision, and ACh had only excitatory effects on the overall activity of the neurons. Figure 9 shows that application of $\mathrm{ACh}$ increased the spontaneous activity and the auditory response. In this case, the tonic response component was strongly increased, whereas the phasic component remained almost stable. Figure 10 shows the effect of different transmitters (GABA, NA, ACh, glutamate) applied on a single neuron. ACh had an excitatory effect similar to glutamate. While the tonic response component was increased, the phasic onset peak was slightly reduced by the action of $\mathrm{ACh}$. This shows that the application of ACh can reduce the height of the onset peak. Similar effects were seen in $37 \%$ of all units, whereas in $60 \%$ the onset peak was unaltered or did increase similarly to the tonic component. Injection of $\mathrm{ACh}$ reduced the latency by 0.04 msec and increased the jitter of latency from 0.469 to 0.483 msec, the significance of these changes, however, was low ( $p=$ 0.82 and 0.5 , respectively, $n=25$ ).

\section{Glutamate}

Glutamate increased both spontaneous and tone-evoked activity (Figs. 10, 12). Onset peak and the tonic component of tone evoked responses and their tonic component were either increased to a similar degree $(63 \%)$, or the tonic component was affected more than the onset peak (21\%) (Table 1; Figs. 10, 12).

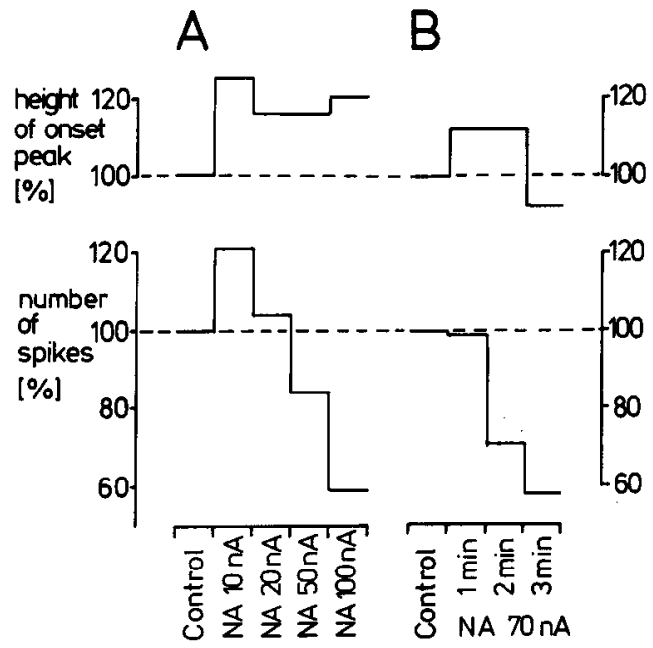

Figure 6. Onset enhancement and changes in the total number of spikes induced by noradrenaline in dependence of injection current $(A)$ and delivery time $(B)$. Top row, relative height of the onset peak derived from PST histograms; lower row, total number of spikes counted during 40 presentations of the acoustic stimulus.

The latency of the first tone-evoked spike was significantly reduced by $0.22 \mathrm{msec}(p>0.99, n=19)$ and the jitter decreased from 0.59 to $0.44 \mathrm{msec}(p>0.99)$. The decrease of jitter was about half of that seen with NA $(0.15 \mathrm{vs} 0.27 \mathrm{msec})$ and was to be expected from the observation that glutamate lowers the threshold to tone stimulation (Fig. 11). The jitter decreased with increasing sound level. In the case of the neuron in Figure 11, prior to glutamate application the jitter amounted to $0.46 \mathrm{msec}$ measured at $60 \mathrm{~dB}$ SPL ( $5 \mathrm{~dB}$ above threshold) compared with $0.13 \mathrm{msec}$ at $80 \mathrm{~dB}$ SPL. Glutamate decreased the jitter at 60 $\mathrm{dB}$ SPL to $37 \mathrm{msec}$ (now about $15 \mathrm{~dB}$ above threshold) and the jitter at $80 \mathrm{~dB}$ SPL to $0.10 \mathrm{msec}$.

In one case, glutamate activated a neuron, recorded in a fully anesthetized animal, which did not respond to tone stimuli at all and was not spontaneously active (Fig. 12). This unit was encountered in close neighborhood to units responding to frequencies around $95 \mathrm{kHz}$. During glutamate application, the unit showed an increasing spontaneous activity and now responded to tone stimuli of $95.7 \mathrm{kHz}$ with a phasic/tonic response pattern. GABA, delivered simultaneously, inhibited both spontaneous and tone-evoked activity, NA suppressed spontaneous activity

Table 1. Effect of different putative transmitters on single units with phasic/tonic response pattern in the anteroventral cochlear nucleus

\begin{tabular}{|c|c|c|c|c|c|c|c|c|}
\hline \multirow[b]{2}{*}{ Variable } & \multicolumn{3}{|l|}{ NA } & \multirow[b]{2}{*}{ GABA } & \multirow[b]{2}{*}{ Glycine } & \multirow[b]{2}{*}{$\mathrm{ACh}$} & \multirow[b]{2}{*}{ Glutamate } & \multirow[b]{2}{*}{ Dopamine } \\
\hline & Awake & Anesthetized & Total & & & & & \\
\hline Total no. of units & 88 & 89 & 177 & 26 & 17 & 35 & 24 & 8 \\
\hline No effect & $9(10 \%)$ & $13(15 \%)$ & $22(12 \%)$ & $3(12 \%)$ & $2(12 \%)$ & $1(3 \%)$ & $3(12 \%)$ & $1(12 \%)$ \\
\hline Pure inhibition & $14(16 \%)$ & $41(46 \%)$ & $55(31 \%)$ & $22(85 \%)$ & $15(88 \%)$ & - & - & $5(62 \%)$ \\
\hline Pure excitation & $8(9 \%)$ & $7(8 \%)$ & $15(9 \%)$ & - & - & $21(60 \%)$ & $15(63 \%)$ & - \\
\hline Selective on-enhancement & $55(63 \%)$ & $27(30 \%)$ & $82(46 \%)$ & $1(4 \%)$ & - & - & $1(4 \%)$ & $2(25 \%)$ \\
\hline
\end{tabular}

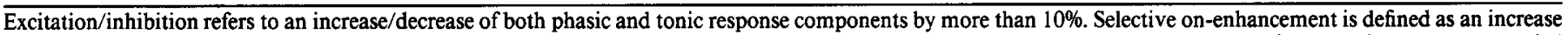

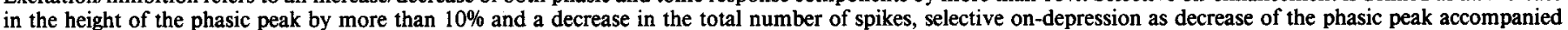

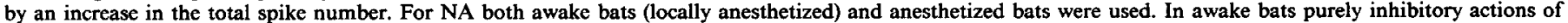
noradrenaline were less frequent and selective on-enhancement was more common than in anesthetized bats. 


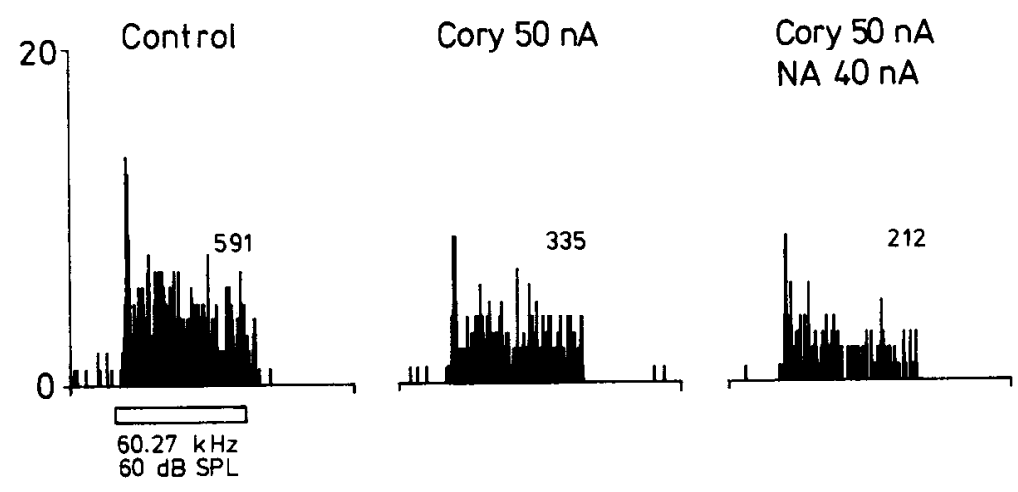

Figure 7. Response patterns (PST histogram) of a single cochlear nucleus unit prior to and during corynanthine (Cory) application, during application of both corynanthine and NA during and after application of NA. The experiments were performed in the order from left to right and top to bottom. Corynanthine depressed both onset peak and tonic response component. The tonic component was suppressed more strongly during simultaneous injection of $\mathrm{NA}$, and the onset peak increases after the cnd of corynanthine application when NA acts alone.

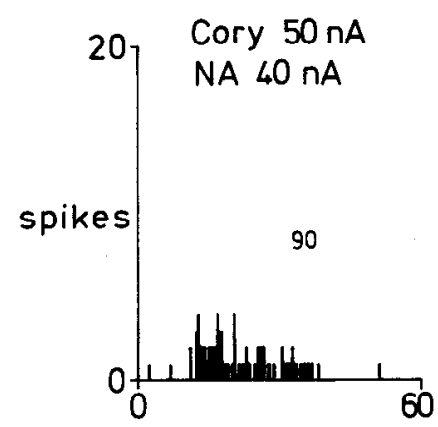

NA $\angle 0$ nA

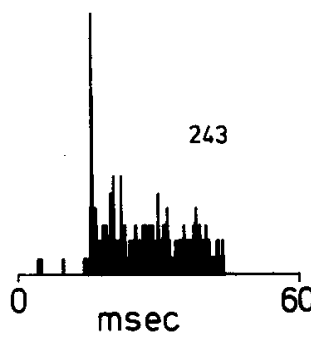

60
Recovery

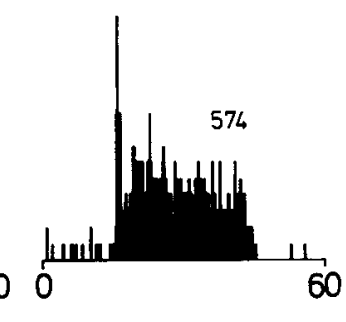

and the tonic component of the auditory response. The phasic component was slightly enhanced. Prolonged application of NA resulted in a strong inhibition similar to the effect of GABA.

\section{Discussion}

\section{Enhancement of auditory temporal contrast}

Among the presumed neurotransmitters in the cochlear nucleus which were tested, only NA could induce selective on-enhancement coupled with tonic inhibition. This points to a specific role of NA in improving auditory temporal contrast. NA is known to enhance sensory responses in the auditory and somatosensory cortex: an enhancement of neuronal activity during the sensory stimulus accompanied by a decrease of the spontaneous activity leads to an improvement of the signal-to-noise ratio (Foote et al., 1975; Woodward et al., 1979; Watcrhouse and Woodward, 1980). The present study confirms these findings and additionally shows that the enhancing action of NA is restricted to phasic neuronal activity at the onset of tone stimuli, whereas tonic auditory response components are inhibited. Consequently, the release of NA should improve the temporal auditory contrast in favor of acoustic transients. This mode of action of NA could be relevant in many behavioral contexts in which the subject is required to analyze new stimuli and to quickly respond to changes in the acoustic environment.

In accordance with this presumed role of NA is the fact that neuronal activity in the locus coeruleus, the main origin of NA fibers, is coupled to orienting behavior and vigilance, situations under which the animal turns its attention towards salient external stimuli (Aston-Jones and Bloom, 1981).

\section{Improvement of timing precision}

Enhancement of the onset component of auditory responses is mainly due to a decrease of the latency jitter of the first spikes evoked by the tone stimulus. Thus, NA improves the temporal precision of coding of auditory events. The distribution of the SDs of the latency in control measurements (Fig. 5) is comparable to data of Young et al. (1988) for primary-like units in the cochlear nucleus of the cat, whereas during application of NA, the average SD decreases, and the distribution becomes more similar to that of cat chopper units (Young et al., 1988). This shows that the temporal characteristics of cochlear nucleus units could be changed to a considerable degree in dependence on the state of activity of the locus coeruleus system and the amount of endogeneously released NA.

Temporal precision is important during passive acoustic orientation and sound localization. The anteroventral cochlear nucleus of Pteronotus provides a major projection to higher brain-stem centers involved in binaural processing (Zook and Casseday, 1985). Temporal cues are an important parameter for the computation of interaural disparities during sound localization (Harnischfeger et al., 1985; Pollak, 1988), which accordingly should benefit from a reduction of temporal jitter in neurons of the anteroventral $\mathrm{CN}$.

In echolocating bats, mechanisms to improve timing precision are of further relevance, since the target distance is measured by the time interval between the response to the emitted echolocation signal and that to the returning echo (Simmons, 1971, 1973). The echolocation call of Pteronotus consists of 4 harmonics with the CF frequencies at about $30,60,90$, and 120 $\mathrm{kHz}\left(\mathrm{CF}_{1-4}\right)$. The response to the emitted call is carried by lowfrequency neurons which are sensitive to the frequency modulated (FM) component of the first harmonic $\left(\mathrm{FM}_{1}\right)$ between 24-30 kHz, whereas the response to the returning echo is coded by neurons tuned to the FM part of higher harmonics $\left(\mathrm{FM}_{2-4}\right)$. In the auditory cortex, neurons responding to input from both the $\mathrm{FM}_{1}$ and $\mathrm{FM}_{2-4}$ frequency range compute the delay between call and echo (O'Neill and Suga, 1979, 1982; Suga and O'Neill, 1979; Suga, 1984). In the cochlear nucleus of Pteronotus, neu- 


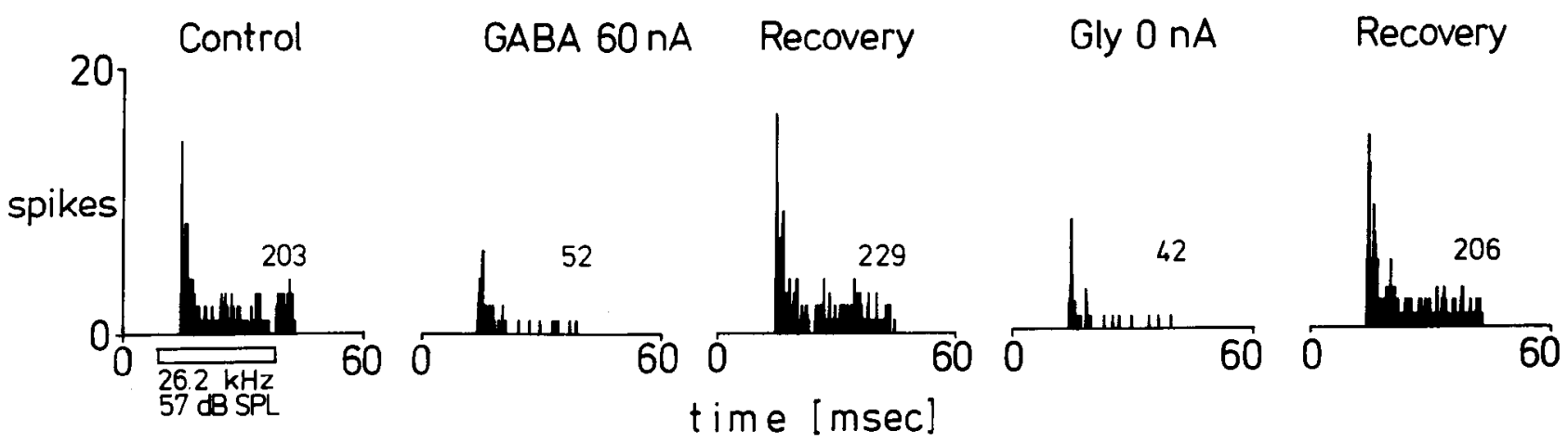

Figure 8. Response patterns (PST histogram) of a single cochlear nucleus unit tested with GABA and glycine. The transmitter substances depress both onset and tonic components of the auditory response. The unit was most sensitive to glycine; the amount of glycine released from the pipette by mere diffusion was enough to clearly affect the auditory response (in control measurements a retention current of $15 \mathrm{nA}$ was applied).

rons sensitive to a wide range of frequencies including $\mathrm{CF}_{1-3}$ and $\mathrm{FM}_{1-4}$ were equally responsive to NA. This indicates that there is no apparent difference in the density of noradrenergic receptors of neurons tuned to frequency ranges where either call or echo are perceived. However, the density of catecholaminergic fibers in the cochlear nucleus of Pteronotus is highest in the marginal cell group and in the most anterior anteroventral subdivision (Kössl et al., 1988), where preferably low frequencies between 24 and $30 \mathrm{kHz}\left(\mathrm{FM} / \mathrm{CF}_{1}\right.$ ) are represented (Kössl, 1987). Thus, low-frequency neurons, carrying the response to the emitted call which is used as a reference for the time delay computation in the auditory cortex, should benefit most from the improvement in temporal precision caused by endogenous NA. An assessment of the amount of endogenously released NA is given by the relative strength of the effects of noradrenergic antagonists. Their effects were indeed most pronounced for neurons tuned to the $F M_{1}$ range. However, because of the difficulty in blocking exogenous NA and the direct effects of the antagonists (see below), this interpretation has to be treated with caution.

\section{Effects of noradrenergic antagonists}

Both propranolol and the $\alpha_{1}$-antagonist corynanthine reduced the onset peak, which indicates that both $\beta$ - and $\alpha_{1}$-receptors could be involved in generating the onset-enhancement induced by NA. The augmenting effect of propranolol on tonic response components was opposite that of NA and further implicates $\beta$-adrenergic mechanisms. Corynanthine and the $\alpha_{2}$-antagonist yohimbine suppressed tonic activity and thus exhibited direct inhibitory effects similar to the results of Howe and Zieglgänsberger (1987) in the dorsal column nuclei. Since $\alpha_{2}$-receptors are located presynaptically, yohimbine actually could potentiate NA release, possibly accounting for the observed suppression. Corynanthine, however, acting on postsynaptic $\alpha_{1}$-receptors, seems to modulate the target neurons directly. Only in a minority of the units tested could propranolol and corynanthine block the enhancing effect of exogenous NA on the onset peak. Only propranolol could antagonize the effect of NA on tonic response components, whereas corynanthine potentiated the suppression of tonic components caused by NA. As discussed by Howe and Zieglgänsberger (1987), the lack of a clear-cut blockade of the effects of exogenous NA and the direct effects of the $\alpha$-antagonists prevent a straightforward interpretation of the results and question the usefulness of these antagonists in extracellular in vivo studies.

\section{Effect of glutamate}

The excitatory effect of iontophoretically applied glutamate in the cochlear nucleus of Pteronotus is comparable to its action in the cochlear nucleus of the chinchilla (Caspary et al., 1981). In most of the units, phasic and tonic response components were increased to a similar degree. In a minority of units $(21 \%)$, glutamate preferentially increased the tonic component. This effect is more pronounced in the chinchilla where application of glutamate can change purely phasic on-responders into purely tonic units (Caspary et al., 1981). Similar to the chinchilla,

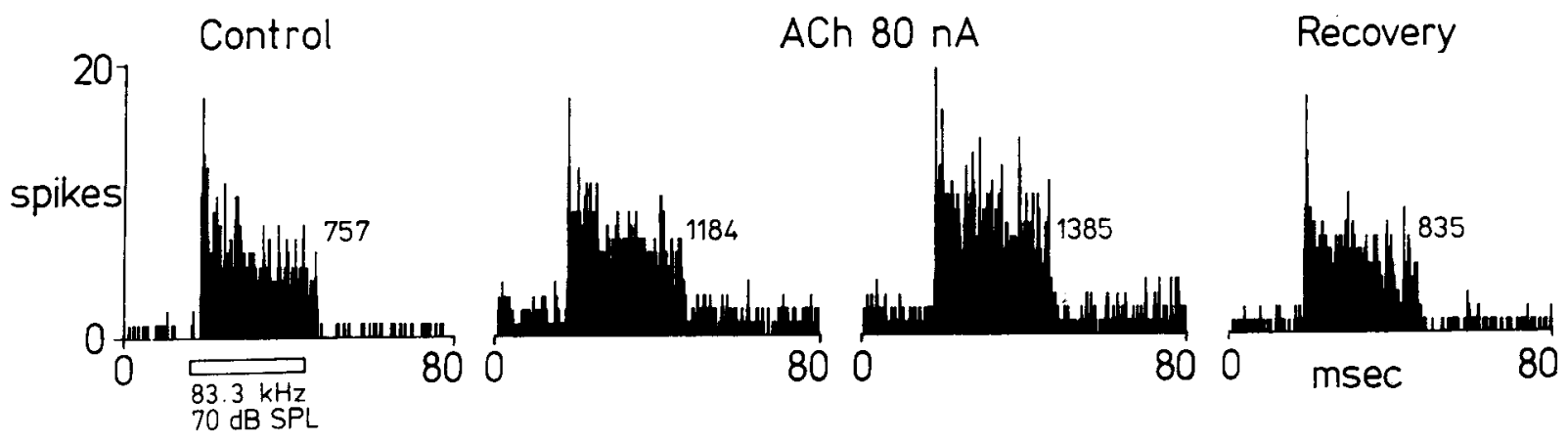

Figure 9. Response patterns (PST histograms) of a single cochlear nucleus unit prior to, during, and after application of ACh. ACh unspecifically increases both the auditory response and spontaneous activity. 

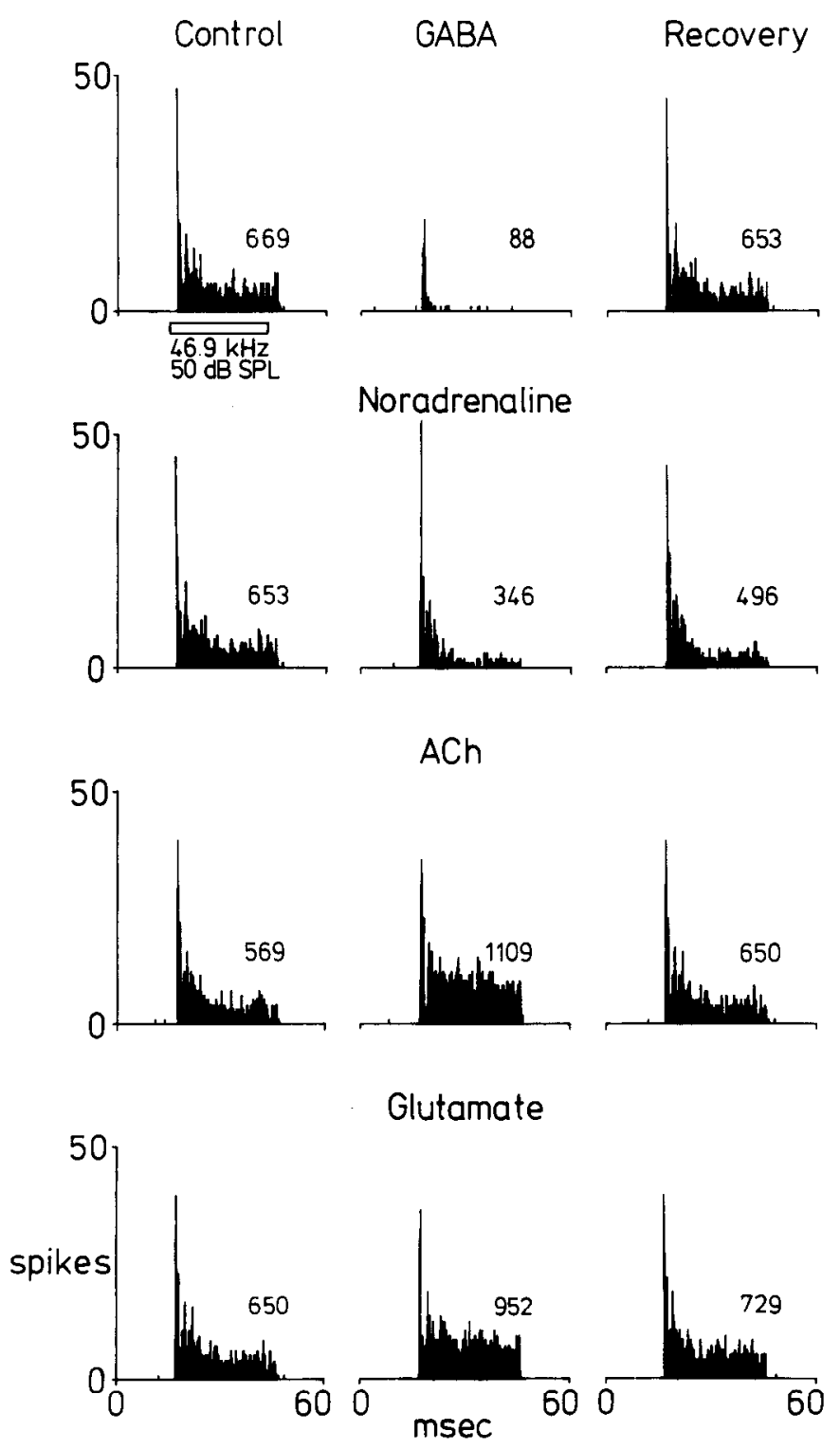

Figure 10. Comparison of the effects of different transmitters on the response patterns of the same single unit (injection current, $70 \mathrm{nA}$ ). The transmitters were applied consecutively starting with the upper row. The recovery measurements for GABA and ACh serve simultaneously as controls for application of the next substance. GABA inhibited all response components, NA enhanced the onset peak and suppressed the tonic response component, and $\mathrm{ACh}$ and glutamate both increased the tonic component while the onset peak decreased slightly.

glutamate is able to lower the auditory threshold of single units, which indicates a glutamate-analogous substance as transmitter of the auditory nerve. This evidence is confirmed by immunocytochemical localization of aspartate aminotransferase in auditory nerve endbulbs (Altschuler et al., 1981), by the demonstration of synaptic release of glutamate and aspartate in the cochlear nucleus (Wenthold, 1979; Canzek and Reubi, 1980; Hansson et al., 1980), and by a decrease in their level after sectioning the auditory nerve (Wenthold and Gulley, 1977).

Both glutamate and NA decreased the jitter of latency. In addition, glutamate also lowered the latency and the auditory threshold of single units which did not change with NA. Since both latency and the jitter of latency decrease with increasing sound level in relation to the threshold (Kitzes et al., 1978), the effect of glutamate on the latency jitter most probably is due to the decrease in threshold and not related to the changes seen with NA.

\section{Effects of GABA and glycine}

In the ventral cochlear nucleus of Pteronotus, both GABA and glycine induced inhibition in a large majority of units and affected onset and tonic response components similarily. The auditory responses of a larger proportion of the total number of units were affected by GABA and glycine than in the chinchilla (Caspary et al., 1979). In Pteronotus, GABA and glycine inhibited 85 and $88 \%$ of the units, in the chinchilla 46 and $70 \%$, respectively. In Pteronotus, the population of neurons which were tested for GABA and glycine exclusively consisted of primary-like and phasic primary-like units, whereas in the chinchilla, response patterns of primary-like neurons were rarely altered by application of the 2 transmitters (Caspary et al., 1979). This indicates that in the ventral cochlear nucleus of Pteronotus there is a larger degree of neuronal inhibition than in a nonspecialized mammal and it remains to be investigated if this is due to echolocation-specific processing.

\section{Effect of $\mathrm{ACh}$}

Modulation of neuronal activity by muscarinic mechanisms causes a slow increase in neuronal excitability (Brown, 1983). Auditory neurons of the chick show this kind of modulation, and both their spontaneous activity and their tone evoked responses are increased by ACh (Müller, 1987). Neuronal activity in the cochlear nucleus of Pteronotus is influenced by $\mathrm{ACh}$ in a similar way. ACh increased both spontaneous and stimulusrelated discharges. In this regard, $\mathrm{ACh}$ acts differently from NA, which only enhances the response activity to tone onset and inhibits all other activity. In contrast to NA, ACh did not decrease but slightly increased the jitter of onset spikes. Both modes of ncuronal modulation should interact in the cochlear nucleus of the mustache bat since both catecholaminergic fibers and fibers associated with $\mathrm{ACh}$-esterase terminate in the same subregions (Kössl et al., 1988). Since neurons are influenced by NA and $\mathrm{ACh}$ in opposite ways, these transmitters should be able to oppose each other functionally and may thus allow for a sophisticated control of neuronal activity in the cochlear nucleus.

\section{References}

Abercrombie, E. D., and B. L. Jacobs (1987) Single-unit response of noradrenergic neurons in the locus coeruleus of freely moving cats. I. Acutely presented stressful and nonstressful stimuli. J. Neurosci. 7: 2837-2843.

Altschuler, R. A., G. R. Neises, G. G. Harmisob, R. J. Wenthold, and J. Fex (1981) Immunocytochemical localization of aspartate aminotransferase immunoreactivity in cochlear nucleus of the guinea pig. Proc. Natl. Acad. Sci. USA 78: 6553-6557.

Aston-Jones, G., and F. E. Bloom (1981) Norepinephrine-containing locus coeruleus neurons in behaving rats exhibit pronounced responses to non-noxious environmental stimuli. J. Neurosci. 1: 887900.

Basile, A. S., and T. V. Dunwiddie (1984) Norepinephrine elicits both excitatory and inhibitory responses in the in vitro rat cerebellar slice. Brain Res. 296: 15-25.

Brown, D. A. (1983) Slow cholinergic excitation-a mechanism for increasing neuronal excitability. Trends Neurosci. 8: 302-307.

Canzek, V., and J. C. Reubi (1980) The effect of cochlear nerve lesions on the release of glutamate, aspartate, and GABA from cat cochlear nucleus, in vitro. Exp. Brain Res. 38: 437-441.

Caspary, D. M., Havey, D. C., and C. L. Faingold (1979) Effects of 


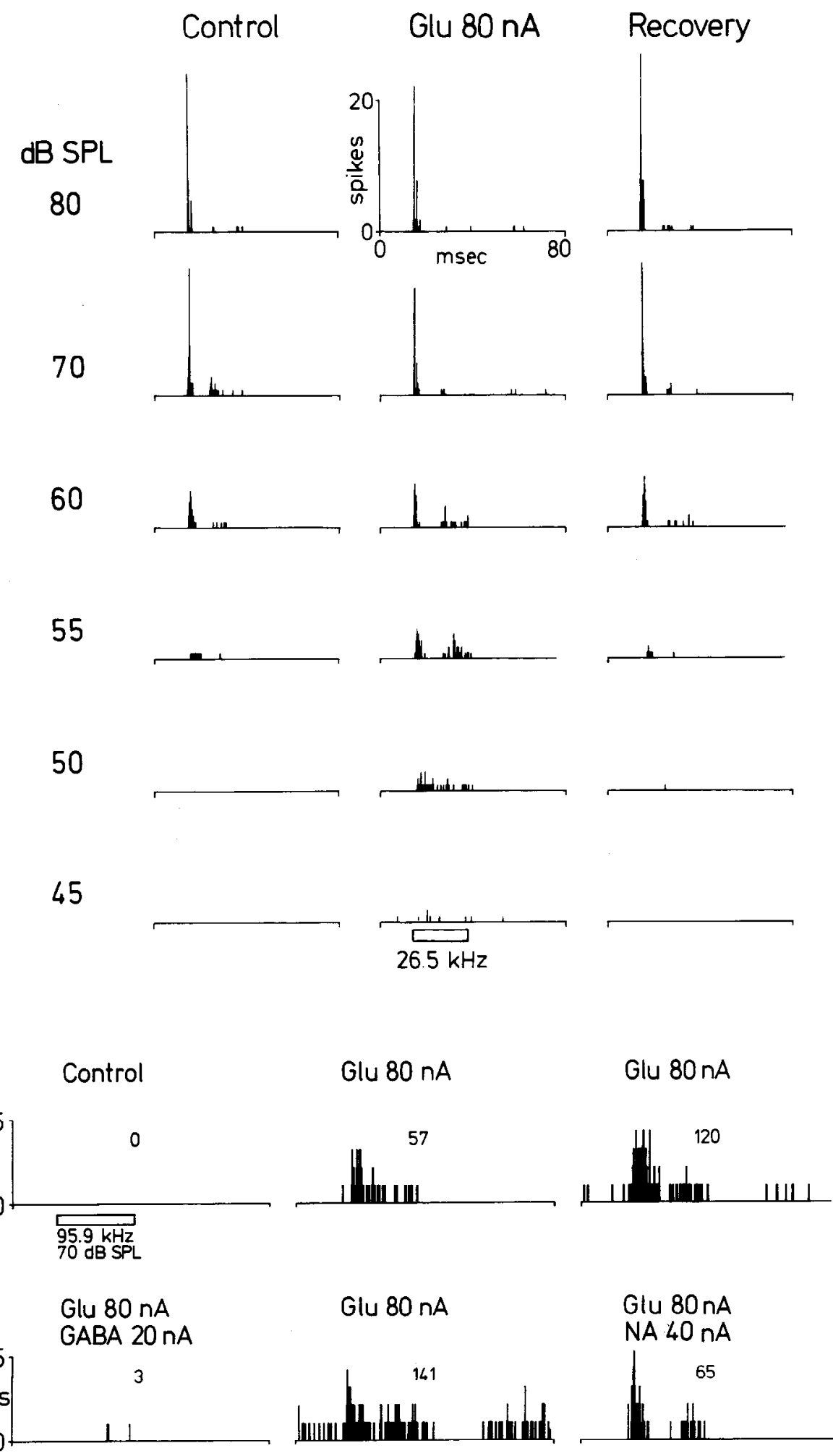

Glu $80 \mathrm{nA}$

NA $40 \mathrm{nA}$

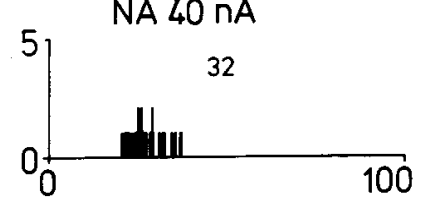

Figure 11. Response patterns of a single cochlear nucleus unit to tone stimuli of different levels prior to (left row), during (middle row), and after application of glutamate (right row). Glutamate lowers the threshold to tone stimulation by about $10 \mathrm{~dB}$.
Figure 12. Glutamate-evoked excitation (PST histograms) of a cochlear nucleus neuron which was not spontaneously active and did not respond to tone stimulation during control measurements (left-most histogram, top row). Only during continuing application of glutamate (starting with the second histogram in the top row), did the neuron respond to sound stimulation and show spontaneous activity. NA, applied additionally, depressed the spontaneous activity and the tonic auditory response, whereas the phasic component was slightly enhanced. Prolonged application of NA resulted in a strong inhibition similar to the effect of GABA. The experiments were performed in the order from left to right and top to bottom. 
microiontophoretically applied glycine and GABA on neuronal response patterns in the cochlear nuclei. Brain Res. 172: 179-185.

Caspary, D. M., Havey, D. C., and C. L. Faingold (1981) Glutamate and aspartate: Alterations of thresholds and response patterns of auditory neurons. Hear. Res. 4: 325-333.

Caspary, D. M., D. C. Havey, and C. L. Faingold (1983) Effects of achetylcholine on cochlear nucleus neurons. Exp. Neurol. 82: 491498.

Collins, G. G. S., G. A. Probett, J. Anson, and N. J. McLaughlin (1984) Excitatory and inhibitory effects of noradrenaline on synaptic transmission in the rat olfactory slice. Brain Res. 294: 211-223.

Feng, A.S., and M. Vater (1985) Functional organization of the cochlear nucleus in rufous horseshoe bats (Rhinolophus rouxi): Frequencies and internal connections are arranged in slabs. J. Comp. Neurol. 235 529-553.

Foote, S. L., R. Freedman, and A. P. Oliver (1975) Effects of putative neurotransmitters on neuronal activity in monkey auditory cortex. Brain Res. 86: 229-242.

Foote, S. L., G. Aston-Jones, and F. E. Bloom (1980) Impulse activity of locus coeruleus neurons in awake rats and monkeys is a function of sensory stimulation and arousal. Proc. Natl. Acad. Sci. USA 77. 3033-3037.

Foote, S. L., F. E. Bloom, and G. Aston-Jones (1983) Nucleus locus ceruleus: Ncw cvidence of anatomical and physiological specificity. Physiol. Rev. 63: 844-914.

Hansson, E., J. Jarlstedt, and A. Sellstrom (1980) Sound-stimulated ${ }^{14} \mathrm{C}$-glutamate release from the nucleus cochlearis. Experientia 36 : 576-577.

Harnischfeger, G., G. Neuweiler, and P. Schlegel (1985) Interaural time and intensity coding in the superior olivary complex and the inferior colliculus of the echolocating bat, Molossus ater. J. Neurophys. 53: 89-109.

Havey, D. C., and D. M. Caspary (1980) A simple technique for constructing "piggyback" multibarrel microelectrodes. Electroenceph. Clin. Neurophysiol. 48: 249-251.

Howe, J. R., and W. Zieglgänsberger (1987) Responses of rat dorsal horn neurons to natural stimulation and to iontophoretically applied noradrenaline. J. Comp. Neurol. 255: 1-17.

Kitzes, L. M., M. M. Gibson, J. E. Rose, and J. E. Hind (1978) Initial discharge latency and threshold considerations for some neurons in the cochlear nucleus complex of the cat. J. Neurophysiol. 41: 11651182.

Kössl, M. (1987) Frequenzrepräsentation und Frequenzverarbeitung in der Cochlea und im Nucleus cochlearis der Schnarrbartfledermaus Pteronotus parnellii. Doctoral Thesis, University of Munich.

Kössl, M., M. Vater, and H. Schweizer (1988) Distribution of catecholamine fibers in the cochlear nuclei of horseshoe bats and mustache bats. J. Comp. Neurol. 269: 523-535.

Malenka, D. A., and R. A. Nicoll (1986) Dopamine decreases the calcium-activated afterhyperpolarization in hippocampal CAl pyramidal cells. Brain Res. 379: 210-215.

McGormick, D. A., and D. A. Prince (1987) Actions of acetylcholine in the guinea-pig and cat medial and lateral geniculate nuclei, in vitro. J. Physiol. (Lond.) 392: 147-165.

Moises, H. C., D. J. Woodward, B. J. Hoffer, and R. Freedman (1979)
Interactions of norepinephrine with Purkinje cell responses to putative amino acid neurotransmitters applied by microiontophoresis. Exp. Neurol. 64: 493-515.

Moore, R. Y., and F. E. Bloom (1979) Central catecholamine neuron systems: Anatomy and physiology of the norepinephrine and epinephrine systems. Annu. Rev. Neurosci. 2: 113-168.

Müller, C. (1987) Differential effects of acetylcholine in the chicken auditory neostriatum and hyperstriatum ventrale-studies in vivo and in vitro. J. Comp. Physiol. A 161: 857-866.

O'Neill, W. E., and N. Suga (1979) Target range-sensitive neurons in the auditory cortex of the mustached bat. Science 203: 69-73.

O'Neill, W. E., and N. Suga (1982) Encoding of target range and its representation in the auditory cortex of the mustached bat. J. Neurosci. 2 : 17-31.

Pollak, G. D. (1988) Time is traded for intensity in the bat's auditory system. Hear. Res. 36: 107-124.

Schnitzler, H.-U. (1970) Echoortung bei der Fledermaus Chilonycteris rubiginosa. Z. Vergl. Physiol. 68: 25-38.

Siegel, S., and N. J. Castellan, Jr. (1988) Nonparametric Statistics for the Behavioral Sciences, McGraw-Hill, New York.

Simmons, J. A. (1971) The sonar receiver of the bat. Ann. NY Acad. Sci. 188: 161-174.

Simmons, J. A. (1973) The resolution of target range by echolocating bats. J. Acoust. Soc. Am. 54: 157-173.

Suga, N. (1984) Neural mechanisms of complex-sound processing for echolocation. Trends Neurosci. 7: 20-27.

Suga, N., and W. E. O'Neill (1979) Neural axis representing target range in the auditory cortex of the mustached bat. Science 206: $351-$ 353.

Waterhouse, B. D., and D. J. Woodward (1980) Interaction of norepinephrine with cerebrocortical activity evoked by stimulation of somatosensory afferent pathways in the rat. Exp. Neurol. 67: 11-34.

Waterhouse, B. D., H. C. Moises, and D. J. Woodward (1980) Noradrenergic modulation of somatosensory cortical neuronal responses to iontophoretically applied putative neurotransmitters. Exp. Neurol. 69: $30-49$.

Wenthold, R. J. (1979) Release of endogenous glutamic acid, aspartic acid and GABA from cochlcar nucleus slices. Brain Res. 162: 338 343.

Wenthold, R. J., and R. L. Gulley (1977) Aspartic acid and glutamic acid levels in the cochlear nucleus after auditory nerve lesion. Brain Res. 138: 11-123.

Woodward, D. J., H. C. Moises, B. D. Waterhouse, B. J. Hoffer, and R. Freedman (1979) Modulatory actions of norepinephrine in the central nervous system. Fed. Proc. 38: 2109-2116.

Young, E. D., J.-M. Robert, and W. P. Shofner (1988) Regularity and latency of units in ventral cochlear nucelus: Implications for unit classification and generation of response properties. J. Neurophysiol. 60: $1-29$

Zook, J. M., and J. H. Casseday (1982) Cytoarchitecture of auditory system in lower brain stem of the mustache bat, Pteronotus parnellii. J. Comp. Neurol. 207: 1-13.

Zook, J. M., and J. H. Casseday (1985) Projections from the cochlear nuclei in the mustache bat, Pteronotus parnellii. J. Comp. Neurol. 237: 307-324. 